\title{
Augmented-reality computed tomography-guided transcatheter pacemaker implantation in dextrocardia and congenitally corrected transposition of great arteries
}

\author{
Maksymilian P. Opolski ${ }^{1}$, Ilona M. Michałowska ${ }^{2}$, Bartosz A. Borucki ${ }^{3}$, \\ Barbara Nicińska $^{4}$, Łukasz Szumowski ${ }^{5}$, Maciej Sterliński ${ }^{5}$ \\ ${ }^{1}$ Department of Interventional Cardiology and Angiology, Institute of Cardiology, Warsaw, Poland \\ ${ }^{2}$ Department of Radiology, Institute of Cardiology, Warsaw, Poland \\ ${ }^{3}$ Interdisciplinary Center for Mathematical and Computational Modelling, \\ University of Warsaw, Poland \\ ${ }^{4}$ Department of Anesthesiology, Institute of Cardiology, Warsaw, Poland \\ ${ }^{5}$ Arrhythmia Department, Institute of Cardiology, Warsaw, Poland
}

A 30-year-old man with congenitally corrected transposition of great arteries and dextrocardia was admitted for leadless transcatheter pacemaker implantation (Micra ${ }^{\mathrm{TM}}$ TPS, Medtronic, $\mathrm{MN}$, USA). At the age of 4 , he underwent patch repair of the ventricular septal defect complicated by complete atrioventricular block. Computed tomography angiography (CTA) using a Somatom Force scanner (Siemens, Erlangen, Germany) was performed for better visualization of the position, orientation and morphology of the subpulmonic ventricle (left ventricular morphology). Three-dimensional CTA reconstructions of the subpulmonic ventricle simulating the angulations of the $\mathrm{C}$-arm in the catheterization laboratory were transmitted to a head-mounted computer (Google Glass, Google Inc, Mountain View, CA) for display in a mobile application equipped with a hands-free voice recognition system developed by the authors (Fig. 1A). The CTA-defined right anterior oblique $60^{\circ}$ view served to guide Micra advancement through the right atrium toward the subpulmonic ventricular apex (Fig. 1B, C), whereas the anterior-posterior projection facilitated lead positioning relative to the lateral orientation of the septum (Fig. 1D, E).

Several technical challenges can be encountered when implanting pacemakers in patients with congenital heart disease and dextrocardia. In the present case, both ventricles were inversed and rotated to the right, and identification of the most optimal stimulation site was restricted due to trabeculation of the subpulmonic ventricle and interventricular patch. Herein, augmented-reality glass was used for display of CTA datasets to define the most optimal fluoroscopic angles and guide pacemaker positioning. This case confirms the utility of augmented-reality technology to optimize complex percutaneous interventions in patients with altered fluoroscopic orientation.

Conflict of interest: None declared

Address for correspondence: Maksymilian P. Opolski, MD, Department of Interventional Cardiology and Angiology, Institute of Cardiology, ul. Alpejska 42, 04-628 Warszawa, Poland, tel: +48 501444303, fax: +48 22 6133819, e-mail: opolski.mp@gmail.com 


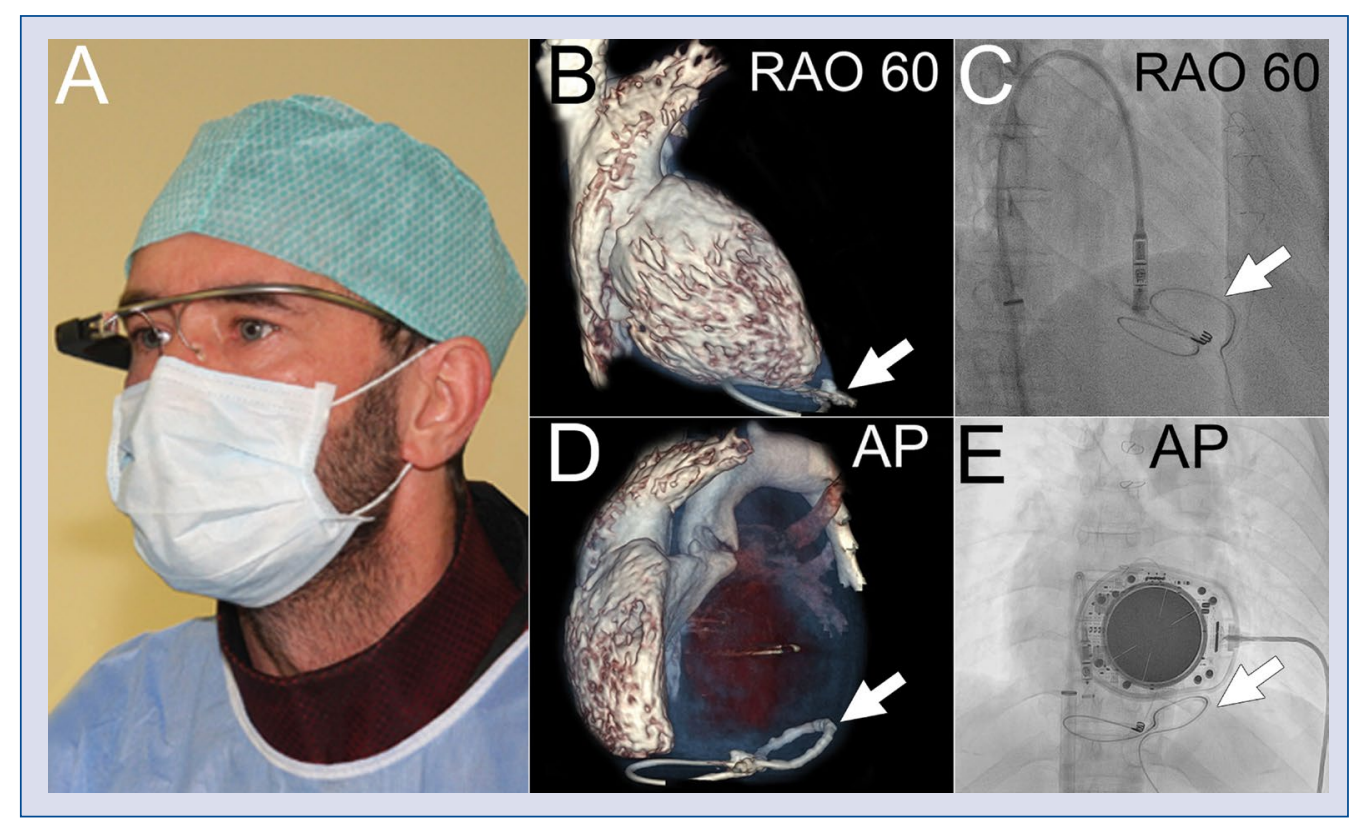

Figure 1. A. Cardiologist viewing the computed tomography (CT) images in the upper right visual field during pacemaker implantation; B, C. Computed tomographic-defined right anterior oblique (RAO) $60^{\circ}$ view with corresponding fluoroscopic projection. The RAO $60^{\circ}$ projection was used to guide the Micra advancement through the right atrium toward the subpulmonic ventricular apex. Note perfect alignment of the contours of the abandoned epicardial lead in CT-generated environment and fluoroscopy (arrows); D, E. Computed tomographic-defined anterior-posterior (AP) view with corresponding fluoroscopic projection. The AP projection facilitated lead positioning within the lower portion of the subpulmonic ventricle. Note perfect alignment of the contours of the abandoned epicardial lead in CT-generated environment and fluoroscopy (arrows). 